\title{
Linx
}

Revue des linguistes de l'université Paris X Nanterre

$12 \mid 2002$

«Comme la lettre dit la vie »

\section{Son non escrit par droite aventure. L'inscription du nom propre dans le Tristan en prose}

Florence Plet

\section{OpenEdition}

\section{Journals}

Édition électronique

URL : http://journals.openedition.org/linx/1309

DOI : 10.4000/linx.1309

ISSN : 2118-9692

Éditeur

Presses universitaires de Paris Nanterre

\section{Édition imprimée}

Date de publication : 1 octobre 2002

Pagination : 231-235

ISSN : 0246-8743

\section{Référence électronique}

Florence Plet, «Son non escrit par droite aventure. L'inscription du nom propre dans le Tristan en prose », Linx [En ligne], 12 | 2002, mis en ligne le 10 octobre 2012, consulté le 19 avril 2019. URL : http://journals.openedition.org/linx/1309; DOI : 10.4000/linx.1309

Ce document a été généré automatiquement le 19 avril 2019

Département de Sciences du langage, Université Paris Ouest 


\title{
Son non escrit par droite aventure. L'inscription du nom propre dans le Tristan en prose
}

\author{
Florence Plet
}

1 Le Tristan en prose ${ }^{1}$, roman de chevalerie du XIII ${ }^{e}$ siècle, rassemble les données de deux ensembles romanesques préexistants : les Tristan en vers du XII siècle et le Lancelot-Graal. Pour le prosateur, les noms propres sont un moyen d'assurer la continuité avec ses prestigieux modèles, mais aussi de produire sa propre matière.

2 Dans une période où le nom est perçu comme intimement lié à son référent, il acquiert volontiers des pouvoirs, sinon vraiment magiques, du moins éminemment romanesques. Par exemple, entendre se nommer un grand chevalier peut faire l'effet d'un coup de massue: "Puis que je oi le non entendu de Lanselot du Lac, je sui ferus de la machue ki ne me laisse la teste lever » $\left(\mathrm{I}, 67_{11}\right)$; plus souvent encore, le nom propre prend l'allure d'un objet qu'on peut manipuler, dont on veut s'emparer et qu'il faut cacher. S'il ne se charge pas exactement de pouvoirs surnaturels, du moins possède-t-il une valeur sociale ${ }^{2}$ ou poétique suffisante pour lui donner quelque puissance agissante.

Or, le nom peut prendre une forme effectivement objectivée par le biais de son inscription matérielle dans la diégèse. Dans la littérature d'origine celtique, au nom écrit s'associent volontiers des effets magiques : ainsi, dans le Lai du Chèvrefeuille de Marie de France, la baguette de coudrier où Tristan a gravé son nom arrête la suite d'Yseut: souvenir, semble-t-il, de légendes irlandaises où le héros fige ses ennemis grâce à un bâton gravé de son nom en ogam ${ }^{3}$. Quant au thème du nom gravé sur (ou sous) la lame d'une tombe, il est éclairé par l'article de R. Colliot, «Les épitaphes arthuriennes ${ }^{4}$ : soit l'inscription est comparable à une geis ${ }^{5}$ qui appelle à la vengeance d'un meurtre ; soit, à la manière des pierres parlantes celtiques ${ }^{6}$, elle légitime un héros comme Lancelot. Dans tous les cas, l'écriture semble conférer au nom un surcroit de merveille.

Quelques passages du Tristan en prose évoquent explicitement le nom sous des formes écrites; elles peuvent se répartir en deux catégories : les inscriptions qualifiantes, qui donnent à Tristan, nouveau héros arthurien, sa légitimité face aux personnages 
canoniques; les épitaphes. Deux pistes retiendront notre attention: quelle part le prosateur fait-il à la merveille dans ces épisodes? Quel rôle jouent-ils dans le roman, en particulier pour ce qui est du démarquage avec l'autre grand héros, Lancelot?

\section{Des inscriptions qualifiantes}

5 Il convenait que Tristan fût mis au rang des héros du Lancelot-Graal. Trois inscriptions le font, placées en des moments stratégiques de l'histoire du héros.

\section{Le perron Merlin}

6 Au moment de confier à Gouvernal le nourrisson Tristan, Merlin lui fait lire des letres entailliees en la pierre ja avoit grant tens passé, gravées de toute éternité, semble-t-il, sur un perron de marbre : «"ci vendront li troi bon chevalier. A ceste fontaine assembleront il (...)" Aprés i avoit letre qui disoient : "Galaaz, Lanceloz, Tristanz" » (C.I, 236) - prédiction de la rencontre, en cet endroit, des trois grands noms encore inconnus, destinés à former la triade de l'excellence.

7 À peine l'histoire personnelle de Tristan s'ouvre-t-elle, que son nom est mis au rang de ses modèles littéraires. "Comme dans la Vulgate, les grandes décisions, qu'elles concernent le destin du héros, le Graal ou Salesbières, ont été prises d'avance par une instance plus ancienne et puissante encore que le prophète des Bretons. Comme dans la Vulgate, dont le Tristan emprunte à de nombreux égards le moule, cette instance a jalonné d'inscriptions et de prophéties la route des personnages »- ainsi R. Trachsler commentet-il cet épisode ${ }^{7}$. D'emblée, au cœur même de sa terre, voici le héros de Cornouailles admis au rang des (futurs) héros de Logres, par le biais d'une merveille proprement arthurienne : une inscription prophétique.

\section{La lettre de Galehaut}

8 La deuxième apparition de nom écrit va dans le même sens. A peine bu le philtre, le couple de Tristan et Yseut reçoit une sorte de reconnaissance de son statut exceptionnel lors de l'épreuve du Château des Pleurs (C.II, 450-465). Sans parler de réécriture, par certains côtés (ne serait-ce que par le nom du château), l'épisode peut se rapprocher de celui de la Douloureuse Garde. Pas de cimetière futur où le héros apprendrait son propre nom, bien sûr. Mais le nom écrit, sur un tout autre support, intervient pour clore le passage: Galehaut écrit à Guenièvre pour l'informer de l'abolition de la mauvaise coutume, récit traité en discours narrativisé. Le prosateur en revanche produit la fin de la lettre : en substance, il existe deux dames en qui «est tote la biauté dou monde, [et deux chevaliers en qui] est tote la chevalerie dou monde. Après [Galehaut]escrit quatre nons: Lancelot dou Lac; Tristan, le neveu le roi Marc de Cornoaille ; la rö̈e Genevre; Yselt la Bloe »(C.II, 482).

Loin d'être « une instance (...) ancienne et puissante », le rédacteur de la lettre, Galehaut, est parfaitement identifié, il n'a rien de magique, et le cadre de la correspondance a peu à voir avec le monument gravé; pourtant, les quatre noms solennellement détachés rappellent la forme de l'inscription du Perron Merlin, qui isolait aussi la triade hors phrase. Quel que soit le support, l'écriture du nom ne se fait pas en toute simplicité : elle suffit à hausser les héros sur un même piédestal. 


\section{Les sièges de la Table ronde} merveilleux, est célèbre ${ }^{8}$. Le Tristan en prose donne à lire cet épisode au tome VI, sous forme d'une interpolation assez fidèle de la Queste : la veille de la Pentecôte, Lancelot et ses cousins constatent que les sièges de la Table ronde comportent tous l'inscription « Cil [=Untel] doit seoir ci » (VI, 93 $\left.{ }_{34}\right)$; aucun nom sur le Siège Périlleux, mais une prédiction: "[demain,] doit cil sieges trouver son maistre " $\left(93_{39}\right)$. Le jour dit, le "nom en blanc" s'actualise, les letres ont changé : "Çou est li sieges Galaad» $\left(101_{35}\right)$. Le jeune homme est invité à s'asseoir en ces termes : «Cil liex est vostres» $\left(101_{38}\right)$. Galaad peut alors tenter l'épreuve du Siège Périlleux.

11 Dans ce passage typique de la Queste, seule la merveille du «nom en blanc » sur le Siège Périlleux est vraiment soulignée : «Ci a merveilleuses aventures!» $\left(93_{41}\right)$. En revanche, l'inscription des noms des chevaliers de la Table ronde n'avait donné lieu à aucun commentaire émerveillé.

12 Le Tristan en prose trouve moyen de combler ce vide au profit de son héros, en inventant l'un des rares prodiges surnaturels de ce roman si rationnel : tous les noms écrits sur les sièges sont réinvestis de merveilleux par l'énonciation d'une coustume establie (III, 271 9 ), pure création', à ma connaissance, du Tristan en prose :

Quant aucuns chevaliers estoit esleüs et apelés a chele hounour par le consentement de tous chiaus de laiens, quant on l'avoit amené au siege ki destinés li estoit, se on n'i trouvoit son non escrit par droite aventure, il estoit du tout refusés (...) et disoient tout conmunaumaument k'il n'estoit pas dingnes de tele hounour rechevoir quant aventure ne s'i acordoit (III, 271 $10^{10}$.

L'honneur est d'autant plus difficile à obtenir, qu'en cas de vacance d'un siège, seul un mieudres chevaliers que chiex ki devant $i$ avoit esté peut l'occuper $\left(272_{12}\right)$; l'énonciation de cette coutume introduit l'intronisation de Tristan: "Li sieges ki jadis fu du Morhaut d'Yrlande li est otroiiés, nous i avom trouvé son non escrit !» $\left(273_{14}\right)^{11}$.

Certes, la validation du nom de Galaad reste célestielle et absolue; le Tristan en prose reconnaît cette donnée de la Queste, par le biais d'une prolepse : à Galaad est réservé d' acompli(r) le Siege Perilleus (III, $272_{14}$ ), et le système d'attribution des sièges vacants à un meilleur chevalier doit cesser avec lui, car après cel boin chevalier n'i peüst venir nul mieudre ne aussi boin. $\left(272_{16}\right)$. Mais le prosateur infuse quelques signes d'élection dans le nom de Tristan : par l'inscription merveilleuse, il est désormais validé, lui aussi, à son niveau, c'est-à-dire de façon mondaine et relative ; meilleur chevalier que son prédécesseur, il peut se lancer officiellement dans la compétition qui caractérise les aventures arthuriennes : la course au renom ${ }^{12}$.

Ces occurrences du nom propre écrit sont comparables aux « épitaphes prophétiques ou messianiques» concernant Lancelot ou Galaad qu'étudie R. Colliot (1973: 163). Les différences sont toutefois notables : en premier lieu, l'élection de Tristan ne s'inscrit pas dans les cimetières, pas plus qu'il ne croise les sépultures de ses ancêtres. Même si son nom le voue à un destin triste, celui-ci ne se décide pas outre-tombe. Au nom caché de Lancelot, répond le nom éclatant de Tristan.

16 D'autre part, ces épitaphes du Lancelot-Graal sont « des textes magiques qui lancent le héros vers un destin exceptionnel et dangereux » (R. Colliot, 1973 : 175). Est-ce le cas des

Linx, 12 | 2002 
inscriptions tristaniennes? Il ne me semble pas qu'elles soient si dynamiques. Elles fixent plutôt le nom (et son référent) dans un certain hiératisme. Il s'agit d'afficher clairement l'objectif du roman : élever Tristan au même niveau que Lancelot, le Tristan en prose au niveau du Lancelot en prose. Enfin, le nom de Tristan est associé au moins une fois à celui d'Yseut dans la lettre.

\section{Les noms sur les tombes}

Le Tristan en prose préfère donc, aux "épitaphes prophétiques ou messianiques ", des monuments aux fonctions proches; en revanche, le roman connaît la catégorie des « épitaphes de vengeance », qui mentionnent le nom de la victime et celui du meurtrier: " consignant les meurtres et poussant au meurtre», ce "sont des textes maléfiques »" Qu'en est-il dans notre roman?

\section{Tombes des victimes de Marc}

Un nom tristement mentionné sur les tombes est celui du roi Marc. Accusé d'avoir assassiné l'un de ses vassaux, Marc occit le plaignant, Armant, en combat public. Lancelot le contraint ensuite à avouer qu'il avait réellement tué son fidèle compagnon. Arthur rétablit alors une justice que le duel judiciaire n'a pas fait ressortir ${ }^{14}$, sous forme d'une épitaphe non reproduite textuellement, mais que le prosateur évoque ainsi : «Et fist li rois Artus escrire sour sa tombe la felonnie le roi March et coment li chevaliers avoit esté mors... » (IV, $90_{56}$ ).

19 Similaire est l'épitaphe de Fairan le Noir, que Marc a voulu empoisonner avec Galaad. Celui-ci, ayant échappé miraculeusement à la mort, fait «escrire letres sus la tombe, qui disoient conment li rois March l'ot ocis par venim »(IX, $\left.27{ }_{49}\right)$.

Quand il s'agit d'évoquer leur sépulture, le nom des victimes - Armant, Fairan - est rayé à la fois du roman et de leur propre tombeau, au profit d'une sinistre mise à l'honneur de Marc. La tombe n'est pas destinée à honorer leur mémoire, mais bien à établir solennellement l'ignominie du meurtrier nommé, lui. Conformes à celles étudiées par R. Colliot, de telles "épitaphes de vengeance " adjugent à ces personnages éphémères un rôle ingrat, mais précis : certes, ils ne déclenchent pas de vendetta particulière, mais ils désignent à la vindicte publique le mauvais roi ; ils ne semblent surgir dans le roman que pour y mourir, et ne valent peut-être que pour leur tombe. Ainsi permettent-ils de graver dans la pierre une vérité intangible : la félonie de Marc est ... monumentale.

\section{Tombes des victimes de Tristan}

21 Curieusement, son neveu Tristan compte également à son passif les épitaphes de deux victimes tombées sous ses coups. Bien sûr, leur valeur est différente. La première, habile invention du prosateur pour infléchir la légende, concerne celle d'Hunesson, mort d'avoir jalousé Tristan ${ }^{15}$. Morgain, en rédigeant le texte funèbre, n'omet pas le nom de son amant, mais il vient après celui du héros :

Elle fist letres entaillier desus la tombe meïsmes, ki disoient : " Tristran, ki ocheïs Huneson ki chi desous gist, saches bien tout chertainnement que sa mors ne fu pas si cruele com la TOIE sera, car TU morras de double mort a grant angousse et a grant martire, et mout aras desiré la mort avant que TU l'aies. Et encore $\mathrm{TE}$ di JEbien une autre cose dont Jouvoeil que TU 
soies chertains : TU ne morras devant che que TU morras de chele meïsmes lanche dont TU L'

ochesis, vraiement le saches TU » (III, $182_{19}$ ).

Prolepse et non prophétie, il faut en convenir. Cependant, on a l'impression que la «valeur programmatique » de l'épitaphe s'inverse ici : l'annonce (extradiégétique) de la mort pourrait venir s'inscrire textuellement sur la sépulture (diégétique). De la même façon s'est inversé le geste de Tristan, qui jadis, de sa main, fit chevalier son écuyer Habé (C.I, $320_{21}, 323_{3}$ ), et qui le tua de cette même main. Mais le lecteur a eu grand temps d'oublier ce nom... D'autant plus que le surnom « le Renommé » contraste avec l'obscurité du personnage. Or, ce surnom était maladroitement justifié dans la prolepse :

et sachiez qu'il fu mout preudons de son cors et de grant renomee, et compaignon de la Table Reonde (...) ; et pour la proesse de li fu il apelez Hebés le Renomez (C.I, $323_{3}{ }_{9}$ ). 
Une dernière tombe enfin s'extrait de ces aller-retour dans le temps: celle de Tristan et d'Yseut. À l'initiative de Marc, leurs corps sont enterrés en la maistre eglise de Tyntajoul (IX, $\left.85_{13}\right)$; la richesse de la sépulture rappelle celle de Galehaut $\left(85_{15}\right)$; il n'y a pas d'épitaphe à proprement parler, mais deux statues grandeur nature, sur lesquelles figurent les noms des amants. "C'est l'ymage de monsigneur Tristran ", "C'est l'ymage de madame la roïne Yseut " ${ }^{18}$. Aucun appel à la vengeance, ici - cela va de soi : Marc le meurtrier est tout de même le maître d'œuvre des tombes! Aucun message non plus. Les noms ne renvoient qu'aux statues, et les statues aux noms ; de fait, est-il besoin de plus ? Le simple fait que les corps des amants soient enfin réunis parle assez. On se rappelle une autre inscription, dans un autre texte: dans le Lai du Chèvrefeuille, Tristan gravait son nom sur une baguette de coudrier (v.54), et cela seul en disait beaucoup plus :

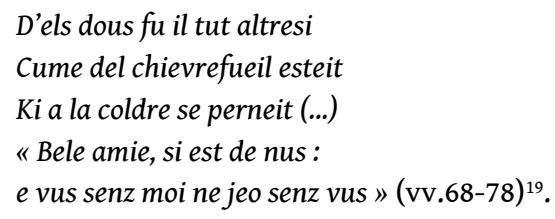

31 C'est bien cette dernière formule qu'entérine le roi Marc en réunissant les amants : Li uns ne pooit demourer sans l'autre, si estoient lour cuers ensamble (...) et pour ce qu.'il s'entramerent tant en lour vie, le fist li rois metre ensamble (IX, 85 6 ).

Les deux corps dans la tombe, les deux statues au pié de cele sepulture $\left(85_{25}\right)$, les deux noms sur les statues. La désincarnation progressive des personnages - corps, puis ymages, letres enfin - suffit à faire entrer dans l'éternité le mythe des amants de Cornouailles. Tant com li siecles duerra sera parlé de cheste mort (IX, $84_{63}$ ). En effet ${ }^{20}$.

Dans la lignée des romans arthuriens, le Tristan en prose sait faire un usage modéré mais significatif de ce thème. Disséminés sur les perrons, sur les sièges de la Table ronde, dans les tombes, ces multiples textes brefs assignent au nom écrit le rôle de mettre le porteur à sa juste place dans ce roman, tout en lui assurant de solides racines dans le corpus arthurien. Pour autant, les inscriptions se démarquent de celles du Lancelot-Graal. Ecrits, les noms de personnes sont le médium efficace d'un pouvoir - pouvoir magique peutêtre: les anciennes geis se profilent confusément, gonflant de mystères séduisants les noms fixés par l'inscription. Mais plus encore certainement, c'est le pouvoir de l'écriture qui ressort : devenu monument, le simple nom propre déploie solennellement l'essentiel de son référent. 


\section{NOTES}

1. Les éditions de référence sont : les trois tomes édités par R.L. Curtis (I, Hueber, Munich, 1963 ; II, Brill, Leiden, 1976 ; III, Arthurian Studies XIV, Woodbridge, D.S. Brewer, 1985), qui donnent le début du roman; les neuf tomes édités sous la direction de Ph. Ménard (Droz, 1987 à 1997). Le tome est indiqué en chiffres romains, suivi du paragraphe et de la ligne en indice; la mention «C.» signale qu'il s'agit de l'édition Curtis.

2. Voir notre article «Incognito et renommée : les innovations du Tristan en prose ", à paraître dans Romania.

3. M. Cagnon, «Chievrefueil and the Ogamic Tradition ", in Romania, XCI, 1970. Ces recherches sont présentées par M. Demaulesdans la notice du Lai du Chèvrefeuille, in Tristan et Yseut. Les premières versions européennes, Ch. Marchello-Niziadir. (Bibliothèque de la Pléiade, Gallimard, 1995), p. 1293.

4. Bulletin Bibliographique de la Société Internationale Arthurienne, 1973, pp. 155-175.

5. Pour simplifier, dans le domaine celtique, une geis est une incantation contraignante. Voir J. Marx, La légende arthurienne et le Graal (PUF, Paris, 1952), p. 77 ; J.R. Reinhard, The survival of geis in medieval romance (Halle, 1933).

6. La Pierre de Fâl intronise par un cri les rois d'Irlande. J. Markale, Nouveau Dictionnaire de mythologie celtique (Pygmalion, Paris, 1999), p. 160, Lia Fail.

7. R. Trachsler, Clôtures du cycle arthurien (Droz, 1996), pp. 154-55.

8. La Queste del saint Graal, A. Pauphiletéd. (Champion, Paris, 1980), pp. $4_{3}$ et $8_{13}$.

9. Autre création, purement pratique et dépourvue de merveilleux : le drap de soie qui sert en quelque sorte à faire l'appel quand Arthur est en déplacement : Estoient escrit ordeneement li non des cevaliers es dras de soie, si que cascuns savoit $u$ il devoit seoir. (...) Et par ce pooit on savoir li quel cevalier $i$ faloient $\left(\mathrm{V}, 182_{24}\right)$.

10. Cela amène d'ailleurs une contradiction : d'après cet épisode du tome III, les sièges seraient nominaux bien avant l'arrivée de Galaad, ... ce qu'oublie le prosateur en interpolant la Queste au tome VI.

11. Sur le Morholt promu chevalier de la Table ronde, voir F. Plet, « Le nom de l'Autre : le géant et le fou dans le roman de Tristan en prose ", in Littératures n³8 (Presses Universitaires du Mirail, Toulouse, printemps 1998), pp. 10-11 ou F. Plet, Les noms propres dans le roman de Tristan en prose (Thèse dactylographiée, Nanterre, 2000), pp. 512-514.

12. Voir article cité note .

13. R. Colliot $(1973: 158,175)$.

14. Voir, à propos de cet épisode, l'introduction de J.C. Faucon, tome IV, p. 40 et E. Baumgartner, La harpe et l'épée ; tradition et renouvellement dans le Tristan en prose (SEDES, 1990), pp. 71-72.

15. Voir R. Trachsler(1996:168-69).

16. R. Colliot (1973: 162).

17. «Du manuscrit trouvé au corps retrouvé ", in Le topos du manuscrit retrouvé, Actes du colloque international de Louvain-Gand, mai 1997 (Louvain-Paris, éditions Peeters, 1999), p. 13.

18. IX, $85_{29}$. Bizarrement, ce paragraphe est répétitif, comme si deux variantes étaient juxtaposées. À partir de la ligne 33, la description de la sépulture recommence; dans cette deuxième version, la statue de Tristan est décrite; les letres sont plus précisément situées : sur le plat de l'épée brandie par Tristan et sur la poitrine d'Yseut; l'inscription est encore plus laconique « Monsigneur Tristran », « Madame Yseut » (IX, 85 ${ }_{47}$ ). 
19. La nature exacte du texte gravé sur le bâton a suscité beaucoup de gloses, que résume utilement M. Demaulesdans la Notice du Lai du Chèvrefeuille, in Tristan et Yseut (Pléiade), pp. 1301-1305.

20. Voir aussi l'interprétation, assez proche, de A.C Van Coolput, «Sur quelques sculptures anthropomorphes dans les romans arthuriens en prose », in Romania ${ }^{\circ} 108,1987$ : « Tout se passe comme si le monument-souvenir tendait vers l'invitation à garder en mémoire ce qu'on doit déchiffrer sur l'image et peut-être aussi, par un mouvement d'expansion,le texte qui enchâsse l'image, en d'autres termes, l'œuvre elle-même » (p. 261).

\section{AUTEUR}

\section{FLORENCE PLET}

Université de Bordeaux 3 